\title{
A symmetry result for strictly convex domains
}

\author{
A. G. Ramm \\ Mathematics Department \\ Kansas State University, Manhattan, KS 66506, USA \\ email: ramm@math.ksu.edu
}

\begin{abstract}
Assume that $D \subset \mathbb{R}^{2}$ is a strictly convex domain with $C^{2}$-smooth boundary.

Theorem. If $\int_{D} e^{i x} y^{n} d x d y=0$ for all sufficiently large $n$, then $D$ is a disc.
\end{abstract}

Key words: Symmetry problems; asymptotic formulas. MSC[2010]: 34E05;

\section{Introduction}

We assume throughout that $D \subset \mathbb{R}^{2}$ is a strictly convex domain and its boundary $S$ is $C^{2}$-smooth. Suppose that

$$
\int_{D} e^{i x} y^{n} d x d y=0, \quad n=0,1,2, \ldots \ldots
$$

Our result is stated as Theorem 1.

Theorem 1. If $D$ is strictly convex bounded domain in $\mathbb{R}^{2}$ and (1) holds, then $D$ is a disc.

This result the author obtained while studying the Pompeiu problem, see, for example, Chapter 11 in the book [2]. The result of Theorem 1 can also be established if the following is assumed in place of equation (11):

$$
\int_{D} e^{i y} x^{n} d x d y=0, \quad n=0,1,2, \ldots \ldots
$$

This follows from the proof of Theorem 1 . 


\section{Proof of Theorem 1.}

Let $\ell$ be an arbitrary unit vector, $L_{1}$ be the support line to $D$ (at the point $s_{1} \in S$ ) parallel to $\ell$, and $L_{2}$ be the support line to $D$ (at the point $q_{1} \in S$ ) parallel to $L_{1}$, where $q_{1}=q_{1}\left(s_{1}\right)$. Since $D$ is strictly convex, one can introduce the equations $y=f(x)$ and $y=g(x)$ of the boundary $S$ between the support points $s_{1}$ and $q_{1}$. For definiteness and without loss of generality let us assume that the orthogonal projection of the point $s_{1}$ onto the line $L_{1}$ lies not lower than the projection of the point $q_{1}$ onto $L_{1}$, and let the $x$-axis pass through $s_{1}$ and be orthogonal to $L_{1}$. The graph of $f$ is located above the graph of $g$. Since $S$ is strictly convex the function $f$ has a unique point of maximum $x_{1}$, where $x_{1} \in(a, b)$, and $f\left(x_{1}\right)>f(x)$ for $x \in[a, b], f\left(x_{1}\right)>0$ and $f^{\prime \prime}\left(x_{1}\right)<0$. Here $a$ and $b$ are the $x$-coordinates of the points $q_{1}$ and $s_{1}, a<b$. Let us denote by $s$ the value of the natural parameter (arc length on $S$ ) corresponding to the maximum point of $f$, that is, to the point $x_{1}$. The function $g$ has a unique point of minimum $x_{2}, x_{2} \in(a, b), g(x)>g\left(x_{2}\right)$, $g\left(x_{2}\right)<0$ and $g^{\prime \prime}\left(x_{2}\right)>0$. From the strict convexity of $S$ it follows that these maximum and minimum are non-degenerate, that is, $f^{\prime \prime}\left(x_{1}\right) \neq 0$, and $g^{\prime \prime}\left(x_{2}\right) \neq 0$. Denote by $q$ the value of the natural parameter corresponding to the minimum point of $g$. Let us write formula (11) as

$$
\int_{D} e^{i x} y^{n} d x d y=\int_{a}^{b} e^{i x} \frac{f^{n+1}(x)-g^{n+1}(x)}{n+1} d x=0, \quad n=0,1,2, \ldots \ldots
$$

The factor $n+1$ in the denominator can be canceled because the integral in (3) equals to zero. We want to take $n \rightarrow \infty$ and use the Laplace method for evaluating the main term of the asymptotic of the integral. Let us recall this known result, the formula for the asymptotic of the integral

$F(\lambda):=\int_{a}^{b} \phi(x) e^{\lambda S(x)} d x=\left(\frac{2 \pi}{\lambda\left|S^{\prime \prime}(\xi)\right|}\right)^{1 / 2} \phi(\xi) e^{\lambda S(\xi)}(1+o(1)), \quad \lambda \rightarrow \infty$,

see, for example, [1. In this formula $\xi \in(a, b)$ is a unique point of a non-degenerate maximum of a real-valued twice continuously differentiable function $S(x)$ on $[a, b], S^{\prime \prime}(\xi)<0$, and $\phi$ is a continuous function on $[a, b]$, possibly complex-valued. We apply this formula with

$$
S(x)=\ln |f|, \quad \lambda:=2 m:=n+1 \rightarrow \infty, \quad \phi=e^{i x},
$$

and take $n=2 m-1$ to ensure that $n+1=2 m$ is an even number, so that $f^{2 m}$ and $g^{2 m}$ are positive, and $\ln f^{2 m}$ and $\ln g^{2 m}$ are well defined. The point 
$x_{2}$ of minimum of $g$ becomes a point of local maximum of the function $g^{2 m}$. Note that $\left|(\ln |f|)^{\prime \prime}\right|=\frac{\left|f^{\prime \prime}\left(x_{1}\right)\right|}{\left|f\left(x_{1}\right)\right|}$ at the point $x_{1}$ where $f^{\prime}\left(x_{1}\right)=0, f\left(x_{1}\right)>0$ and $f^{\prime \prime}\left(x_{1}\right)<0$.

Taking the above into consideration, one obtains from (3) the following asymptotic formula:

$$
\begin{array}{r}
\int_{D} e^{i x} y^{n} d x d y=\left[e^{i x_{1}+2 m \ln \left|f\left(x_{1}\right)\right|}\left(\frac{\pi\left|f\left(x_{1}\right)\right|}{m\left|f^{\prime \prime}\left(x_{1}\right)\right|}\right)^{1 / 2}-\right. \\
\left.e^{i x_{2}+2 m \ln \left|g\left(x_{2}\right)\right|}\left(\frac{\pi\left|g\left(x_{2}\right)\right|}{m\left|g^{\prime \prime}\left(x_{2}\right)\right|}\right)^{1 / 2}\right](1+o(1))=0, \quad n \rightarrow \infty,
\end{array}
$$

where $2 m=n+1, x_{1} \in(a, b)$ and $x_{2} \in(a, b)$. It follows from the above formula that the expression in the brackets, that is, the main term of the asymptotic, must vanish for all sufficiently large $m$. This implies that $f\left(x_{1}\right)=\left|f\left(x_{1}\right)\right|=\left|g\left(x_{2}\right)\right|$ and $\left|f^{\prime \prime}\left(x_{1}\right)\right|=g^{\prime \prime}\left(x_{2}\right)=\left|g^{\prime \prime}\left(x_{2}\right)\right|$, because $f\left(x_{1}\right)>0, g\left(x_{2}\right)<0, f^{\prime \prime}\left(x_{1}\right)<0$ and $g^{\prime \prime}\left(x_{2}\right)>0$. It also follows from formula (5) that $e^{i x_{1}}=e^{i x_{2}}$. This implies $x_{1}=x_{2}+2 \pi p$, where $p$ is an integer. The integer $p$ does not depend on $s$ because $p$ is locally continuous and cannot have jumps. Thus,

$$
x_{1}-x_{2}:=2 \pi p ; \quad\left|f\left(x_{1}\right)\right|=\left|g\left(x_{2}\right)\right| ; \quad\left|f^{\prime \prime}\left(x_{1}\right)\right|=\left|g^{\prime \prime}\left(x_{2}\right)\right| .
$$

We prove in Lemma 2 (see below) that $p=0$. Another proof of this is given in the Remark 1 below the proof of Lemma 2.

Consider the support lines $L_{3}$ at the point $s$ and $L_{4}$ at the point $q$, where $L_{3}$ and $L_{4}$ are orthogonal to $\ell$. Denote by $L=L(s)$ the distance between $L_{3}$ and $L_{4}$, that is, the width of $D$ in the direction parallel to $\ell$. Note that $L=f\left(x_{1}\right)-g\left(x_{2}\right)>0$, and

$$
L=(r(s)-r(q), \ell),
$$

where $r=r(s)$ is the radius vector (position vector) corresponding to the point on $S$ which is defined by the parameter $s$. This point will be called point $s$. The same letter $s$ is used for the point $s \in S$ and for the corresponding natural parameter. Let $R=R(s)$ denote the radius of curvature of the curve $S$ at the point $s$ and let $\kappa=\kappa(s)$ denote the curvature of $S$ at this point. Then one has

$$
R^{-1}=\kappa=\left|f^{\prime \prime}\left(x_{1}\right)\right|
$$

because $\kappa=\left|f^{\prime \prime}\left(x_{1}\right)\right|\left[1+\left|f^{\prime}\left(x_{1}\right)\right|^{2}\right]^{-\frac{3}{2}}$ and $f^{\prime}\left(x_{1}\right)=0$ since $x_{1}$ is a point of maximum of $f$. 
From (6) we will derive that

$$
L(s)=2 R(s), \quad \forall s \in S .
$$

It will be proved in Lemma 2, see below, that equation (9) implies that $D$ is a disc. Thus, the conclusion of Theorem 1 will be established.

We denoted by $r=r(s)$ the equation of $S$, where $s$ is the natural parameter on $S$ and $r$ is the radius vector of the point on $S$, corresponding to $s$. One has $r^{\prime}(s)=t$, where $t=t(s)$ is a unit vector tangential to $S$ at the point $s$. We have chosen $s$ so that $t(s)$ is orthogonal to $\ell$. Since $\ell$ is arbitrary, the point $s \in S$ is arbitrary. The point $q \in S, q=q(s)$, is uniquely determined by the requirement that $t(q)=-t(s)$, because $S$ is strictly convex. One has $(r(s)-r(q), \ell)=L$, where $L=L(s)$ is the width of $D$ in the direction parallel to $\ell$. Since $r^{\prime}(s)=t(s)$, the first formula (6) implies

$$
\left(r(q)-r(s), r^{\prime}(s)\right)=2 \pi p, \quad\left(r(q)-r(s), r^{\prime}(q)\right)=-2 \pi p, \quad \forall s \in S .
$$

Differentiate the first equation (10) with respect to $s$ and get

$$
\left(r^{\prime}(q) \frac{d q}{d s}-r^{\prime}(s), r^{\prime}(s)\right)+\left(r(q)-r(s), r^{\prime \prime}(s)\right)=0, \quad \forall s \in S .
$$

Note that $r^{\prime}(s)=t(s)=-t(q)=-r^{\prime}(q)$ and $r^{\prime \prime}(s)=\kappa(s) \nu(s)$, where $\nu(s)$ is the unit normal to $S$ (at the point corresponding to $s$ ) directed into $D$, and $(r(s)-r(q), \ell)=L(s)=(r(q)-r(s), \nu(s))$, because $\nu(s)$ is directed along $-\ell$. Consequently, it follows from (11) that

$$
-\frac{d q}{d s}-1+\kappa(s) L(s)=0, \quad \forall s \in S .
$$

One has $L(s)=L(q)$, and it follows from formulas ([6) that $\kappa(s)=\kappa(q)$.

Differentiate the second equation (10) with respect to $q$ and get

$$
\left(t(q)-t(s) \frac{d s}{d q}, t(q)\right)+\left(r(q)-r(s), r^{\prime \prime}(q)\right)=0 .
$$

Note that $t(q)=-t(s)$ and $r^{\prime \prime}(q)=\kappa(q) \nu(q)$, where $\nu(q)=-\nu(s)$ because $L_{3}$ is parallel to $L_{4}$. Consequently, equation (13) implies

$$
\frac{d s}{d q}+1-\kappa(s) L(s)=0, \quad \forall s \in S
$$

Compare (12) and (14) and get $\frac{d s}{d q}=\frac{d q}{d s}$. Thus, $\left(\frac{d q}{d s}\right)^{2}=1$. Since $\frac{d q}{d s}>0$, it follows that

$$
\frac{d s}{d q}=\frac{d q}{d s}=1, \quad \forall s \in S
$$


Therefore, equation (12) implies

$$
\kappa(s) L(s)=2 \quad \forall s \in S .
$$

Let us derive from (16) that $D$ is a disc.

Recall that $s$ is the natural parameter on $S, L(s)$ is the width of $D$ at the point $s$ (that is the distance between two parallel supporting lines to $S$ one of which passes through the point $s)$ and $\kappa(s)$ is the curvature of $S$ at the point $s$.

Lemma 2. Assume that $D$ is strictly convex domain with a smooth boundary $S$. If equation (16) holds, then $D$ is a disc.

Proof of Lemma 2. Denote by $K$ the maximal disc inscribed in the strictly convex domain $D$, and by $r$ the radius of $K$. If there are no points of $S$ outside $K$, then $D$ is a disc and we are done. If $S$ contains points outside $K$, let $x \in S$ be such a point. Consider the line $\tilde{L}$ passing through the center of $K$ and through the point $x \in S, x \notin K$. Let $L^{\prime}$ be the support line to $S$ orthogonal to the line $\tilde{L}$ and tangent to $S$ at a point $x^{\prime}, x^{\prime} \notin K$. Denote the radius of curvature of $S$ at the point $x^{\prime}$ by $\rho$. One has $\rho \leq r$, because $K$ is the maximal disc inscribed in $D$. The width $L$ of $D$ at the point $x^{\prime}$ in the direction of the line $\tilde{L}$ is greater than $2 r$ because $x^{\prime} \notin K$. One has $L>2 r$ and $L=2 \rho \leq 2 r$. This is a contradiction. It proves that $D=K$. Thus, $D$ is a disc, and, consequently, the parameter $p$ in formula (6) is equal to zero. Lemma 2 is proved.

Thus, Theorem 1 is proved.

Remark 1. Let us give another proof that $p=0$, where $p$ is defined in formula (6). One has $L(s)=(r(q)-r(s), \nu(s))$. Differentiate this equation with respect to $s$ and get

$$
L^{\prime}(s)=-(r(q)-r(s), \kappa(s) t(s))+\left(r^{\prime}(q) \frac{d q}{d s}-r^{\prime}(s), \nu(s)\right),
$$

where $t(s)$ is the unit vector tangential to $S$ at the point $s$. Here the known formula $\nu(s)^{\prime}=-\kappa(s) t(s)$ was used. The second term in the formula (17) vanishes since $r^{\prime}(s)$ and $r^{\prime}(q)$ are orthogonal to $\nu$. Thus, $L^{\prime}(s)=-2 \pi p \kappa(s)$. Since $D$ is strictly convex, one has inequality $\min _{s \in S} \kappa(s) \geq \kappa_{0}>0$, where $\kappa_{0}>0$ is a constant. The function $L(s)$ must be periodic, with the period equal to the arc length of $S$. The differential equation $L^{\prime}(s)=-2 \pi p \kappa(s)$ does not have periodic solutions unless $p=0$. Therefore, $p=0$. 


\section{References}

[1] Bleistein, N. and Handelsman, R., Asymptotic expansions of integrals, Dover Publications, New York, 1986.

[2] Ramm, A. G., Inverse Problems, Springer, New York, 2005. 